\title{
ESTUDO DESCRITIVO SOBRE AS CARACTERÍSTICAS DEFINIDORAS DO DIAGNÓSTICO DE ENFERMAGEM: DISFUNÇÃO SEXUAL
}

\author{
Isabel Cristina Fonseca da Cruz*l \\ Roselena Bazilli Bergamasco** \\ Maria Aparecida de Jesus Belli** \\ Emilia Saito** \\ Luiza Akiko Komura Hoga* \\ Rita de Cássia Tapié Martins** \\ Lucia Cristina Florentino** \\ Isilia Aparecida Silva*
}

CRUZ, I.C.F. da et al. Estudo descritivo sobre as características definidoras do diagnóstico de enfermagem: disfunçāo sexual. Rev. Esc. Enf. USP, v. 26, n.1, p. 43-64, mar. 1992.

As características do diagnóstico de enfermagem de disfunção sexual foram obtidas por meio da análise de 96 históricos, entre 450, cadastrados no Núcleo de Assistência ao Autocuidado da Mulher (NAAM). As 9 características definidoras propostas pela North American Nursing Diagnoses Association (NANDA) foram utilizadas como referencial para a coleta de dados. As características mais frequentes foram: falta de desejo sexual (71.9\%), corrimento (64.5\%) e dor (44.8\%). Conclui-se que das nove, seis características definidoras não apareceram; que a falta de desejo e o corrimento podem ser considerados como características menores (freqüência entre 50 e $70 \%$ ) para a difunção sexual em mulheres. Recomenda-se a realização de novos estudos junto a clientela e seus parceiros para clarificar a presença das demais caracteristicas não detectadas.

UNITERMOS: Diagnóstico de enfermagem. Taxonomia. Disfunção Sexual.

\section{INTRODUÇÃO}

Geralmente quando se fala em autocuidado é comum pensar-se na realização das atividades diárias de vida, tais como: escovar os dentes, fazer higiene corporal, pentear-se e outras.

Todavia o autocuidado, enquanto um referencial teórico, extrapola as atividades físicas e rotineiras, compreendendo também as atividades efetuadas pelos indivíduos em outras áreas.

Durante a realização de uma disciplina de pós-graduação, intitulada “ Autocuidado na Enfermagem", foi-nos solicitada uma pesquisa que abordasse aspectos referentes a prática profissional baseada no Autocuidado.

\footnotetext{
* Doutorandas da Escola de Enfermagem da Universidade de Sāo Paulo - Sāo Paulo.

* Mestrandas da Escola de Enfermagem da Universidade de Sāo Paulo - São Paulo.
} 
Procurando sair do lugar comum, fomos buscar dados sobre a prática de autocuidado no padrão de resposta humana referente ao estabelecimento de vínculos e relaçōes (CRUZ; CRUZ, 1990), mais especificamente no campo da sexualidade.

Por acreditarmos que esta é uma área onde podem ser feitos diagnósticos e tratamentos de enfermagem, decidimos eleger uma categoria diagnóstica específica, proposta pela North American Nursing Diagnosis Association (NANDA), e confrontar as suas características com as referidas pela clientela do NAAM (Núcleo de Assistência ao Autocuidado da Mulher).

Conhecer profundamente um diagnóstico, suas características e os fatores relacionados a sua causa, é um processo que necessariamente deve anteceder a elaboração do plano terapêutico de enfermagem. Os diagnósticos comuns a um grupo de indivíduos delineiam o perfil deste grupo, permitindo um direcionamento global das intervenções de enfermagem.

Assim, apresentamos neste estudo uma análise retrospectiva do histórico de enfermagem do NAAM, particularmente daqueles que apresentaram registros referentes às características definidoras do diagnósticos de disfunção sexual.

Verificamos também, neste trabalho, a freqüência das características definidoras do diagnóstico de disfunçâo sexual na população estudada.

\section{REFERENCIAL CONCEITUAL}

A Disfunção Sexual como diagnóstico de enfermagem.

O diagnóstico de enfermagem é resultante de um julgamento clínico a partir da coleta de dados realizada durante o histórico, sobre as relações do indivíduo, família ou comunidade, às doenças ou processos de vida. CRUZ (1990) ressalta que, uma vez elaborado, o diagnóstico serve de base para as prescriçōes de enfermagem necessárias a sua resolução.

Visando estabelecer uma terminologia universal sobre diagnósticos de enfermagem, foi criada na América do Norte (Estados Unidos e Canadá) uma associação, North American Nursing Diagnosis Association (NANDA), cujo objetivo é coordenar as pesquisas nesta área. Esta associação, define assim o diagnóstico (TAYLOR一LOUGHRAN, 1990):

“O diagnóstico de enfermagem é um julgamento clínico sobre as respostas do indivíduo, família ou comunidade aos problemas de saúde/processos de vida, reais ou potenciais. Os diagnósticos de enfermagem proporcionam a base para a seleção das intervenções de enfermagem necessárias ao alcance dos resultados pelos quais o enfermeiro é responsável."

As características definidoras (CD) de um diagnóstico sāo os dados clíni$\cos$ (sinais, sintomas ou fatores de risco) que validam a presença de uma categoria diagnóstica. A partir de 1986, a NANDA, para fins de pesquisa, classificou as $\mathrm{CD}$ em maior ou menor, visando determinar futuramente quais os sinais e sintomas críticos para a validação de um diagnóstico. Uma CD é considerada maior quando está presente em 80 a $100 \%$ das pessoas ou grupo com o diagnóstico. Uma CD é menor quạndo está presente em 50 a $79 \%$ da população estudada (CARPENITO, 1989). 
Segundo a autora, os fatores relacionados (FR) a um diagnóstico referemse às situações clínicas ou pessoais que podem alterar o estado de saúde ou influenciar no desenvolvimento do problema. De modo geral, a importância do fator relacionado, quando conhecido, reside no fato de que para ele será dirigida a intervenção de enfermagem. Estes não serão objeto de estudo neste trabalho.

Segundo a NANDA (1989) a disfunção sexual é um "estado no qual o indivíduo vive uma mudança na sua função sexual que é vista como insatisfatória, não compensadora e inadequada."

Dentro da Taxonomia I (NANDA, 1989) este diagnóstico, de $\mathbf{n}^{2}$ 3.2.1.2.1., pertence ao Relacionamento, que é um dos 9 Padrões de Resposta Humana. Segundo CRUZ; CRUZ (1990), o Relacionamento refere-se a capacidade da pessoa em estabelecer vínculos com os seus semelhantes. Este padrão compreende as respostas relativas à socialização, ao desempenho de papel e à sexualidade.

As características definidoras da disfunção sexual são ainda segundo a NANDA (1989): "verbalização do problema, alterações (subjetivas) no alcance do papel sexual, limitação real ou subjetiva imposta por doença ou terapia, conflitos envolvendo valores, alteração no alcance da satisfação sexual, inabilidade para alcançar a satisfação desejada, procura de confirmação da desejabilidade, alteração na relação com a pessoa significativa, mudança de interesse em si e nos outros."

Os fatores relacionados a disfunção sexual, conforme a NANDA (1989), são: "alteração bio-psico-social da sexualidade, modelos ineficazes ou ausentes, abuso físico, abuso psico-social, isto é, relaçōes traumáticas, vulnerabilidade, conflito de valores, perda da privacidade, perda da pessoa significativa, alteração na estrutura corporal ou função (gravidez, puerpério, drogas, cirurgia, anomalias, processo de doença, trauma, radiação), desinformação ou falta de conhecimento."

Segundo SUPLICY (1983), o número de problemas sexuais que tem causa orgânica não chega a $10 \%$. Nas mulheres, as queixas referem-se: à falta de desejo primário (nunca ter sentido o erótico ou nunca ter se masturbado), à falta de atração por outro (devido a conflitos edipianos não resolvidos), à estimulação inadequada (medo de engravidar, marido rápido), à dificuldade em se comunicar sexualmente, à raiva ou ressentimento na relação e, principalmente, ao medo da intimidade. Sobre isso, a autora acrescenta que ter intimidade significa ter a capacidade de cuidar do outro, responsabilidade, confiança e livre comunicação sobre os sentimentos.

\section{METODOLOGIA}

O Núcleo de Assistência ao Autocuidado da Mulher (NAAM).

Desde 1986, o Núcleo de Assistência para o Autocuidado da Mulher (NAAM), vinculado ao Departamento de Enfermagem Médico-Cirúrgica da Escola de Enfermagem da USP, vem prestando assistência às mulheres que procuram a Unidade Básica de Saúde de Vargem Grande Paulista (UBS-VGP). Composto por docentes, enfermeiros e graduandos, o NAAM realiza consultas com base na teoria de déficit de autocuidado (OREM, 1985).

Na consulta de enfermagem do NAAM é utilizado o instrumento denomi- 
nado Histórico da Saúde da Mulher, englobando: o histórico de enfermagem autoaplicado (HEAA), a entrevista e o exame físico (ANEXO).

A validação do HEAA está firmada no trabalho de CIANCIARULLO (1975) que o define como sendo "uma série de perguntas organizadas com o objetivo de levantar dados significativos para a enfermeira sobre o paciente, cujas respostas são preenchidas pelo próprio paciente sem assistência direta ou orientação da pesquisadora'.

Neste nosso estudo, a filosofia adotada pelo NAAM, operacionalizada pelo histórico auto-aplicado, nos permite levantar possíveis categorias diagnósticas de enfermagem nesta clientela visto que os dados se baseiam num sólido referencial de enfermagem.

Tipo de pesquisa

A pesquisa tem um caráter exploratório e descritivo, utilizando para isto a auditoria retrospectiva do histórico auto-aplicado.

A população do estudo

Compreende 450 clientes que possuem o histórico auto-aplicado arquivado no banco de dados do NAAM.

A amostra

É composta por 96 clientes que responderam " não" à pergunta $n^{\mathfrak{Q}} 28$ : “ $\mathrm{A}$ senhora fica satisfeita após as relações sexuais?"'. 0

Instrumento da pesquisa

Para a coleta das características definidoras do diagnósticos de disfunção sexual, utilizou-se um formulário, cuja validação baseou-se na literatura (NANDA, 1989)

Este formulário buscava dados, presentes nos históricos arquivados, a partir da análise das seguintes perguntas:

Quadro I - Dados de interesse para análise retrospectiva nos Históricos de Saúde da Mulher - NAAM. São Paulo, 1990.

$\begin{array}{lllllllllllllllllllll}\text { perguntas } & 2 & 4 & 5 & 6 & 7 & 8 & 11 & 12 & 13 & 17 & 22 & 23 & 24 & 25 & 26 & 27 & 28 & 29 & 33 & 39\end{array}$ dados

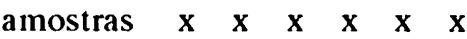

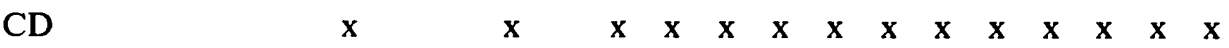

A coleta de dados

Os dados foram coletados pelas autoras em consulta direta aos 96 históricos do NAAM, selecionados conforme o critério anteriomente explicitado. 


\section{A análise de dados}

Os dados do histórico sobre disfunção sexual foram analisados conforıne sua frequêencia relativa.

\section{DISCUSSÃO DOS RESULTADOS}

\section{A caracterização da amostra}

A amostra constitui-se de $50(52.08 \%)$ mulheres brancas, 36 (37.50\%) pardas, $08(8.34 \%)$ pretas, $01(1.04 \%)$ amarela e $01(1.04 \%)$ sem declarar a cor, perfazendo um total de $96(100.0 \%)$ mulheres que relataram não ter satisfação após as relações sexuais.

A resposta sobre insatisfação sexual sugere uma possível disfunção orgásmica. Caberia uma investigação mais detalhada durante a consulta no sentido de determinar a causa desta disfunção. Cabe entretanto ressaltar que, de acordo com MUNJACK; OZIEL (1984), a anorgasmia coital é uma variante normal da resposta sexual feminina, pois mesmo uma mulher que não seja neuroticamente inibida possui um limiar orgásmico elevado, não sendo capaz de atingir o clímax apenas através do coito.

A disfunção sexual e as caracteríticas definidoras

Os dados referentes a característica relativas à verbalização do problema foram extraídos do HEAA nas questões $n^{2} 27$ e 28 .

Tábela 1: Distribuição da manifestação do desejo sexual enquanto característica definidora do diagnósticos de disfunção sexual. São Paulo, 1990

\begin{tabular}{lll}
\hline desejo sexual & $\mathrm{N}$ & $\%$ \\
\hline Sim & 13 & 13.5 \\
Não & 69 & 71.9 \\
Às vezes & 14 & 14.6 \\
\hline Total & 96 & 100.0 \\
\hline
\end{tabular}

Os dados descritos na Tabela 1 demonstram que a evidência " falta de desejo" pode ser entendida como uma característica menor, pois aparece em $71.9 \%$ da população.

Sobre esta freqüência elevada, entendemos que, diante de algumas características desta clientela, como por exemplo: baixa instrução (apenas 10 clientes possuem o $1^{\circ}$ grau completo) e predomínio de atividades domésticas, deve-se considerar no contexto da assistência componentes como tradição cultural, restri- 
ções sociais e religiosas as quais, segundo BELFORT (1986) concedem somento aos homens êxito no desejo e satisfação, condenando a mulher à inferioridade e submissão.

Não é possível todavia apenas através do histórico caracterizar se a disfunção sexual é de natureza primária, quando o individuo nunca experimentou uma resposta adequada, ou não. Isto porque, a disfunção sexual pode estar ralacionada a um dado momento, evento ou pessoa específica.

Os desvios da saúde sexual ocorrem como resultado da interação comple$x a$ entre o indivíduo e o ambiente. Geralmente os distúrbios do desejo sexual provocam na pessoa uma ansiedade mais profunda e intensa, merecendo maior atenção por parte do profissional.

A "limitação real ou subjetiva imposta por doenças ou terapia" enquanto característica definidora, teve relação no HEAA com as questões $\mathbf{n}^{2} 13,17,23$, $24,29,39$. Na parte II, entrevista, os dados foram coletadas nas questōes $n^{2} 12$, 13 e 14; na parte III, exame físico, foram coletados dados referentes a pressão arterial e ao sistema genito-urinário.

Tabela 2: Distribuição das limitaçōes como característica definidora do diagnóstico de disfunção sexual. São Paulo, 1990

\begin{tabular}{|c|c|c|c|c|}
\hline I imitacōes & $\begin{array}{c}\operatorname{sim} \\
\mathbf{N} \\
\%\end{array}$ & $\begin{array}{c}\text { não } \\
\mathbf{N} \\
\%\end{array}$ & $\begin{array}{c}\text { às vezes } \\
\mathbf{N} \\
\%\end{array}$ & $\begin{array}{c}\text { total } \\
\mathbf{N} \\
\%\end{array}$ \\
\hline \multirow[t]{2}{*}{ dispaurenia } & 43 & 37 & 16 & 96 \\
\hline & 44.8 & 38.5 & 16.7 & 100.0 \\
\hline \multirow[t]{2}{*}{ sangramento } & 05 & 83 & 08 & 96 \\
\hline & 05.4 & 86.5 & 08.3 & 100.0 \\
\hline \multirow[t]{2}{*}{ gravidez } & 01 & 95 & I & 96 \\
\hline & 01.0 & 99.0 & I & 100.0 \\
\hline \multirow[t]{2}{*}{ corrimento } & 62 & 34 & I & 96 \\
\hline & 64.5 & 35.4 & I & 100.0 \\
\hline \multirow[t]{2}{*}{ menopausa } & 03 & 93 & 03.12 & \\
\hline & 03.1 & 96.9 & 1 & 100.0 \\
\hline
\end{tabular}

Dos dados descritos na Tabela 2, o corrimento aparece com uma frequiência superior a $50 \%$, classificando-o assim como uma característica menor para a categoria diagnóstica Disfunção Sexual.

A incidência de corrimento em $64.5 \%$ da população estudada sugere-nos o possível diagnóstico de Déficit de Conhecimento sobre higiene da genitália externa, relacionado à falta de informação. 
Quanto a dor, os dados mostram uma frequiência significativa (44.8\%). A dor pode particularmente comprometer a fase do desejo sexual e pode também estar associada a alguma patologia do sistema reprodutivo (MUNJACK; OZIEL, 1984). De igual modo, as queixas de sangramento, presente em $5.4 \%$ da amostra, devem ser investigadas.

Tabela 3: Métodos contraceptivos utilizados pelas clientes com possível diagnósticos de disfunção sexual. São Paulo, 1990.

\begin{tabular}{lll}
\hline & $\mathrm{N}$ & $\%$ \\
$\begin{array}{l}\text { métodos } \\
\text { contraceptivos }\end{array}$ & \\
\hline homornal & 45 & 46.87 \\
barreira & 08 & 08.33 \\
laqueadura & 08 & 08.33 \\
coito interrompido & 02 & 02.08 \\
nenhum & 16 & 16.67 \\
outros* & 17 & 17.70 \\
\hline Total & 96 & 100.0 \\
\hline
\end{tabular}

* outros: DIU, chá, etc.

Qualquer condição que afete o perfil hormonal necessário, como por exemplo: contraceptivos hormonais, menopausa é outros, pode ter influência no desejo sexual. De acordo com MUNJACK et al. (1984), as pesquisas sobre o efeito de contraceptivos orais (utilizado por $46.87 \%$ da população estudada) sobre a libido da mulher não são conclusivas.

Das afirmações a respeito dos contraceptivos hormonais, podemos relacionar as que dizem respeito a diminuição da libido devido a instalação de um quadro depressivo (PIATO, 1984). Em contrapartida, HATCHER (1983) a firma haver um aumento da libido nas mulheres que fazem o uso de contraceptivos hormonais, por conta do afastamento do medo de engravidar.

Analisando os métodos contraceptivos usados no Brasil PINOTTI (1988), referindo-se a laqueadura, considera que a possibilidade de transtornos menstruais e alterações na função ovariana é discutível pois, os estudos realizados chegam a conclusões conflitantes.

Quanto ao coito interrompido, método presente em $2.08 \%$ da população estudada nesta pesquisa, entendemos que esta estratégia impede a entrega total dos parceiros, levando com o passar do tempo a uma diminuição da libido de ambas as partes. 
Para que o método contraceptivo escolhido possa ser entendido como característica definidora do diagnóstico de disfunção sexual, são necessários estudos exploratórios junto a clientela a fim de que se possa avaliar melhor a influência destes fatores na geração do problema, tendo em vista a freqüência inferior a $50 \%$ no grupo amostral.

Quadro II: Distribuição das patologias referidas pelas clientes com possível diagnóstico de disfunção sexual. São Paulo, 1990.

\begin{tabular}{lll}
\hline & patologias & N \\
\hline \multirow{2}{*}{ distúrbios } & gênito-urinário & 87 \\
& PAD> =90mmHg & 27 \\
PAS> = 140mmHg & 17 \\
hipertensão & 13 \\
diabetes & 02 \\
distúrbios mental & 02 \\
& asma & 01 \\
\hline
\end{tabular}

Obs: Neste quadro cabe mais de uma resposta.

No Quadro II aparece tanto hipertensão quanto valores pressóricos acima de determinados limites porque nem sempre coincidia a informação da cliente com a do profissional. Assim sendo, as autoras optaram por colocar ambos os registros de forma que hipertensão refere-se à informação dada pela cliente e valores pressóricos, aos dados do exame físico. De igual maneira tratamos dos "problemas genito-urinário", agrupando nesta categoria tanto as queixas quanto os dados do exame físico, independentemente de coincidências.

Pelo Quadro II, verifica-se que a maioria das mulheres deste estudo (90.6\%) apresentavam problemas gênito-urinários dos quais destacamos a leucorréia, a cistocele, a retocele e a rotura perineal.

Uma descarga vaginal aumentada pode comprometer a atividade sexual, causando desconforto à mulher e interferindo muitas vezes nos jogos sexuais. Além do que a terapêutica da leucorréia pode implicar em abstinência temporária.

A existência de rotura perineal (ou a experiência de múltiplos partos por via baixa) pode também relacionar-se à disfunção porque muitos homens e mulheres, segundo MUNJACK; OZIEL (1984), fantasiam que uma vagina estreita é condição necessária à obtenção do prazer sexual máximo. Ainda segundo este autor (1984) a clistocele e a retocele volumosa também levam as clientes a se queixar de falta de sensação decorrente da vagina extemamente frouxa.

Durante as consultas, $28.1 \%$ das mulheres apresentaram uma pressão arterial diastólica maior ou igual a $90 \mathrm{mmHg}$. Embora uma única verificação não seja conclusiva para a deteç̧ão de um hipertenso, vale chamar atenção para tal fato. 
De acordo com MUNJACK; OZIEL (1984), o ato sexual não parece constituir um risco sério para o cliente hipertenso. Mas, no nosso entendimento a hipertensão não detectada ou não tratada pode comprometer também a saúde sexual da pessoa. A freqüência significatica da pressão diastólica aumentada no grupo estudado sugere uma ligação entre os dois fenômenos.

Em nosso estudo, $2.1 \%$ das mulheres referiram ter diabetes. $O$ diabetes melitus é uma patologia que pode esta associada à disfunção sexual, alterando a potência orgásmica em 1/3 das mulheres diabéticas, segundo BRUNNER; SUDDARTH (1990).

Quanto aos distúrbios mentais presentes em $2.1 \%$ das clientes, não se pode distinguir, pela análise do histórico, se os mesmos tinham uma natureza depressiva ou maníaca. Os casos depressivos perdem o interesse sexual, enquanto que nos maníacos há aumento do impulso sexual (MUNJACK; OZIEL 1984).

\section{CONCLUSĀO}

A finalidade do desenvolvimento dos diagnósticos de enfermagem é clarificar a pratica entre os enfermeiros e outros profissionais. Neste sentido, no que toca ao diagnóstico de disfunção sexual, vimos que numa população de 450 clientes atendidas pelo NAAM, $96(21.33 \%)$ mulheres apresentaram a possibilidade de tê-lo imputado.

Das características definidoras apontadas pela NANDA (1989), para esta categoria diagnóstica, foram identificadas como características menores a falta de desejo, presente em $71.9 \%$ das clientes, e o corrimento (64.5\%).

A dor teve uma freqüência significativa (44.8\%), devendo ser explorada a sua presença em estudos posteriores para que se possa incluí-la ou excluí-la como característica definidora.

Diante de freqüência de $28.1 \%$ de clientes com valores pressóricos diastólicos superiores a $90 \mathrm{mmHg}$, entendemos ser importante desenvolver junto a esta clientela um programa de deteç̧ão, tratamento e controle da hipertensão arterial.

Cabe ressaltar que o histórico de enfermagem possui limitações referentes a exploração de problemas desta natureza, sendo inclusive difícil detectar através da auditoria características subjetivas como alteração no alcance do papel sexual, conflito de valores, alteração no alcance da satisfação sexual, inabilidade em alcançar a satisfação, procura de confirmação da desejabilidade, alteração com pessoa significativa e mudança de interesse em si e nos outros.

Neste sentido, faz-se necessária a realização de novos estudos que busquem junto a esta clientela as características definidoras que não estão presentes no histórico.

Sugerimos inclusive que os dados sejam coletados também junto aos parceiros. Na medida do possível, deve-se levantar também na comunidade, da qual as clientes fazem parte, a concepção da mesma sobre sexualidade.

CRUZ, I.C.F. da el al. Descriptive study: defining characteristics of the nursing diagnosis: sexual disfunction. Rev. Esc. Enf. USP, v. 26, n. 1, p. 43-64, mar. 1992. 
The defining characteristics of the nursing diagnosis "Sexual Dysfunction"' were gathered from NAAM assessesment records. From this population 96 records with the defining characteristics of Sexaul Dysfuntion were selected. The 9 defining characteristics proposed by The North American Nursing Diagnosis Association were used as a framework for the data collection tool. The 3 most frequently difining characteristics were absence of sexual desire (71.9\%), vaginal discharge (64.5\%), and pain (44.8\%). The authors concluded that 6 defining characteristics have not apperead; absence of sexual desire and vaginal discharge can be considered as minor characteristics. The authors recommend the implentation of other studies to clarify the subjectif defining characteristics.

UNITERMS: Nursing diagnosis. Taxonomy. Sexual dysfunction.

\section{REFERÊNCIAS BIBIOGRÁFICAS}

BELFORT, P. Orgasmo feminino: meta terapêutica e realização pessoal. In: VITIELO, N (org.) Sexologia II. São Paulo, Roca, 1986.

BRUMMER, L.S; SUDDARTH, D.S. Tratado de enfermagem médico-cirúrgica, $6^{\circ}$ ed. Rio de Janeiro, Guanabara Koogan, 1990, v.1, cap. 13, p. 172-87: Sexualidade humana.

CARPENITO, L.J. Nursing diagnosis: apllication to clinical practice. 3 ed. Philadelphia, J.B. Lip-pincott, 1989.

CIANCIARULLO, T.I. Histórico de enfermagem auto aplicado: estudo sobre sua utilização em pacientes hospitalizados. São Paulo, 1975. 243p. Dissertaçāo (Mestrado) - Escola de Enfermagem da Universidade de São Paulo.

CRUZ, I.C.F. da Diagnóstico de enfermagem e sua aplicação: revisāo da literatura. Rev. Esc. Enf. USP, v. 24, n. 1, p. 149-62, 1990.

CRUZ, I.C.F. da; CRUZ, D. de A.L.M. Os padrōes de comportamento humanos: uma proposta de traduçāo da Taxonomia I sobre os diagnósticos de enfermagem.Rev. Esc. Enf. USP, v. 25, n. 1, p. 16-19, 1991.

HATCHER, R.A. et al. Planejamento familiar. Rio de Janeiro, Ao Livro Técnico, 1983.

MUNJACK, D.J.; OZIEL, L.J. Sexologia: diagnóstico e tratamento. São Paulo, Atheneu, 1984.

NORTH AMERICAN NURSING DIAGNOSIS ASSOCIATION Taxonomy I: revised with official diagnostic categories. Saint Louis, 1989.

OREM, D.E. Nursing concepts of practice. New York, McGraw-Hill, 1985.

PIATO, S. Anticoncepcionais. Clin Med, v. 2, n. 5, p. 32-8, 1984.

PINOTTI, J.A. et al. Uma análise crítica da contracepçāo no Brasil: parte I. Feminina, v. 16, n. 7, p. $564-90,1988$.

SUPLICY, M. Conversando sobre sexo. Petrópolis. Vozes. 1983.

TAYLOR-LOUGHRAN, A.E. Toward a nursing diagnosis definition. Clin Nurse Special, v. 4, n. 2, p. 71-5, 1990. 


\section{ANEXO I}

DATA:

ÁREA:

PARTE I

\section{'HISTÓRICO AUTO-APLICADO'}

data de nasc.

01 - NOME:

\section{FICHA FAMILIA}

RG:

02 - OCUPAÇÃO:

03 - ENDEREÇO:

PONTO DE REFERÊNCIA:

04 - IDADE

- Menos de 15 anos

- De 15 a 25 anos

- De 26 a 35 anos

- De 36 a 45 anos

- De 46 a 55 anos

- De 56 a 65 anos

- Mais de 66 anos

05 - COR

- Branca

- Parda

- Amarela

- Preta 
06 - ESTADO CIVIL

- Solteira

- Casada

- Desquitada

- Separada Judicialmente

- Divorciada

- Separada

- Viúva

- Amasiada (vive c/ companheiro)

07 - QUAL SUA RELIGIÃO?

08 - GRAU DE INSTRUÇÃO (ESTUDO)

- Primeiro grau completo

incompleto

Que série:

- Segundo grau completo

incompleto

Que série:

- Universitário completo

incompleto

Que série:

- Analfabeto

09 - QUANDO DESCERAM AS PRIMEIRAS REGRAS? (INCÔMODO)

- Antes dos 10 anos

- Entre 10 e 12 anos

- Entre 12 e 15 anos

- Após 15 anos

- Nunca apresentou

10 - DE QUANTO EM QUANTO TEMPO APARECEM OU APARECIAM AS REGRAS?

- Uma vez entre 20 e 25 dias

- Uma vez entre 26 e 30 dias

- Uma vez entre 31 e 35 dias

- Uma vez mais de 36 dias

- De modo irregular 
11 - QUANTOS DIAS COSTUMA FICAR COM AS REGRAS?

- Até 3 dias

- De 3 a 6 dias

- De 6 a 10 dias

- Mais de 10 dias

12 - QUANDO FORAM SUAS ÚLTIMAS REGRAS?

- HÁ menos de 1 mês

- Há mais de 1 mês

- Há mais de 2 meses

- Há mais de 4 meses

- Há mais de 6 meses

- Há mais de 1 ano

- Há mais de 2 anos

- Há mais de 5 anos

- Há mais de 10 anos

13 - A SENHORA ACHA QUE ESTÁ GRÁVIDA ATUALMENTE?

$-\operatorname{Sim}(\quad)$

Não

14 - A SENHORA APRESENTOU ALGUM SANGRAMENTO POR BAIXO, FORA DAS REGRAS, NESTES ÚLTIMOS TEMPOS?

$-\operatorname{Sim}(\quad)$

- Não me lembro

Não

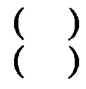

15 - QUANDO?

- Há alguns dias

- Há uma semana

- Há algumas semanas

- Há 1 mês

- Há mais de 1 mês

— Há alguns meses

16 - QUANTAS VEZES?

- Uma vez

- Duas vezes

- Três vezes

- Mais de três vezes

17 - A SENHORA TEM ALGUM TIPO DE CORRIMENTO?

$-\operatorname{Sim}(\quad)$

Não ( )

18 - HÁ QUANTO TEMPO?

— Há 1 mês

- Há mais de 2 meses

- Há mais de 4 meses 
- Há mais de 6 meses

- Há 1 ano

19 - DE QUE COR É O CORRIMENTO?

- Branco

- Amarelo

- Transparente

- Esverdeado

- Com sangue

- Outra cor

Qual?

20 - TEM CHEIRO?

$-\operatorname{Sim}()$

Não ( )

21 - TEM SENTIDO COCEIRA NAS PARTES DE BAIXO?

$-\operatorname{Sim}(\quad)$

Não ( )

22 - COM QUE IDADE A SENHORA TEVE SUA PRIMEIRA RELAÇÃO SEXUAL?

- Antes dos 15 anos

- Entre os 15 e 18 anos

- Após 18 anos

23 - SENTE DOR DURANTE OU APÓS UMA RELAÇÃO SEXUAL?

- Sim

- Não

- Às vezes

24 - TEM SANGRAMENTO DURANTE OU APÓS UMA RELAÇÃO SEXUAL?

- Sim

- Não

- Às vezes

25 - QUAL O NÚMERO DE VEZES QUE A SENHORA COSTUMA TER RELAÇÕES SEXUAIS?

- várias vezes ao dia

- 2 a 3 vezes por semana

- 1 vez por semana

- 1 vez em cada 15 dias

-1 vez em mais de 15 dias 
26 - QUANTOS COMPANHEIROS A SENHORA JÁ TEVE?
- Um
- Três
- Mais de cinco
()
()
— Dois

27 - A SENHORA SENTE VONTADE DE TER RELAÇÕES SEXUAIS?

- Sim
- Não
- Às vezes

28 - A SENHORA FICA SATISFEITA APÓS AS RELAÇŌES SEXUAIS?

- Sim

- Não

- Às vezes

29 - A SENHORA USA ALGUM DESTES MÉTODOS PARA EVITAR FILHOS?

- Pílulas

- Diafragma

- DIU

- Geléias

- Protetor (camisinhas)

- Nenhuma das anteriores

— Outros métodos - Quais?

30 - DURANTE QUANTO TEMPO USA OU USOU ANTI-CONCEPCIONAIS?

- Menos de 1 ano

- Um ano

- Dois anos

- Três anos

- Quatro anos

- Cinco anos

- Mais de cinco anos

31 - A SENHORA JÁ ESTEVE GRÁVIDA ALGUMA VEZ?

- Sim

- Não

- Não sei

32 - QUANTAS VEZES?

— Uma 
$\begin{array}{lllll}\text { - Três } & ( & \text { - Oito } & ( \\ \text { - Quatro } & ( & \text { - Nove } & ( \\ \text { - Cinco } & ( & \text { - Mais de nove } & (\text { ) }\end{array}$

33 - QUANTOS FILHOS A SENHORA TEVE?

- Nenhum
- Um
- Dois
- Três
- Quatro

- Cinco

- Seis

- Sete

- Oito

- Mais de Oito

34 - QUE TIPOS DE PARTO A SENHORA TEVE?

- Normal

- Cesariana

- Fórceps

35 - COM QUE IDADE TEVE SEU PRIMEIRO FILHO?

- Antes dos 15 anos

- Entre 15 e 18 anos

- Entre 18 e 30 anos

- Após os 30 anos

36 - JÁ PROVOCOU ALGUM ABORTO?

$-\operatorname{Sim}()$

Não （）

37 - QUANTOS?
- Um
- Dois

38 - COMO FEZ O ABORTO?

39 - A SENHORA OU ALGUÉm DA SUA FAMÍlIA TEVE OU TEM ALGUMA DESSAS DOENÇAS?

- Diabetes

quem

- Pressão alta

$\operatorname{Sim}$

quem

— Problemas do coração

Sim

Sim

( ) Não

Não

quem 
- Problemas mental

Sim

Não

quem

- Câncer

Sim

Não

quem

— Outras. Quais?

quem

40 - ALGUÉM DA SUA FAMÍlIA MORREU POR ALGUMAS DESSAS DOENÇAS?

- Quem

- Qual doença

41 - A SENHORA TRABALHOU FORA DE SUA CASA? (CASO "NÃO", NÃO PRECISA RESPONDER ÀS DEMAIS QUESTÕES)

$-\operatorname{Sim}()$

42 - QUANTAS HORAS A SENHORA TRABALHA POR DIA?

- Menos de 6 horas

- De 6 a 8 horas

- Mais de 8 horas

43 - O QUE QUE A SENHORA ACHA DO SEU TRABALHO?

- Está satisfeita

- Sente-se pressionada

- Feliz

- Pretende mudar

- Não gosta

44 - A SENHORA ESTÁ EXPOSTA NO SEU TRABALHO?

- À umidade

- À poeira

- À radiação

- À fumaça

- À produtos químicos

- Ao frio

- Ao calor

- Nenhuma das anteriores

OBSER VAÇÕES: 
HISTÓRICO DE SAÚDE DA MULHER

ENTREVISTA

PARTE II

NOME:

RG: FICHA FAMILIA:

01 - QUAL FOI O MOTIVO DA ÚlTIMA CONSULTA MÉDICA?

02 - QUAL O MOTIVO DA CONSULTA ATUAL?

03 - CITE OS PROBLEMAS DE SAÚDE QUE A SENHORA RESOLVE SOZINHA?

04 - COMO COSTUMA RESOLVER ESTES PROBLEMAS?

05 - QUANDO TEM PROBLEMAS DE SAÚDE, ALÉM DO MÉDICO, QUAIS AS PESSOAS QUE VOCÊ PROCURA? 
06 - VOCÊ SENTE ALGUMA CIOSA DURANTE AS REGRAS? O QUÊ?

07 - O QUE VOCÊ FAZ PARA SE SENTIR MELHOR NESSAS OCASIÕES?

08 - O QUE VOCÊ ACHA QUE SÃO AS REGRAS?

09 - JÁ FEZ ALGUM TRATAMENTO PARA DESCER AS REGRAS?

10 - VOCÊ UTILIZA ALGUM TRATAMENTO PARA O CORRIMENTO? QUAL?

11 - O QUE A SENHORA ACHA DO SANGRAMENTO FORA DAS REGRAS?

12 - A SENHORA JÁ APRESENTOU ALGUMA DOENÇA VENÉREA? FEZ TRATAMENTO? 
13 - A SENHORA UTILIZA DROGAS? QUAIS?

14 - A SENHORA TEM O HÁBITO DE BEBER OU FUMAR?

15 - CONDIÇÕES ATUAIS DE SAÚDE:

- Repouso:

- Sono:

- Atividade física:

— Hidratação:

— Alimentação:

- Higiene:

16 - QUEIXAS OBSERVAÇŌES: 


\section{HISTÓRICO DE SAÚDE DA MULHER}

\section{PARTE III}

'EXAME FÍSICO'

NOME:

RG:

FICHA FAMÍLIA:

SINAIS VITAIS:

$\mathrm{T}=$ $\mathrm{PA}=$

CABEÇA/PESCOÇO: (Gânglios, dor, tumoração)

MAMAS:

- Aspecto da pele

- Dor:

- Nódulos:

- Secreção:

- Outras alterações:

AUTO-EXAME DE MAMAS:

- Conhecimento, época do ciclo, mamilos:

- Abdômen:

\section{SISTEMA GENITO-URINÁRIO}

- Queixas:
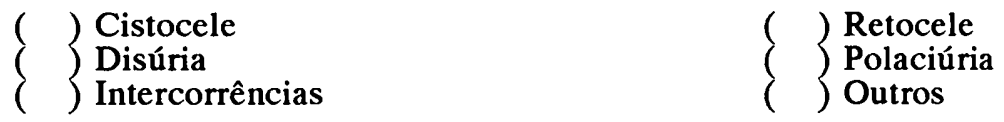

Quais? 
- Paredes vaginais:

\section{SECREÇÕES:}

- Aspecto:

- Quantidade:

- Odor:

- Cor:

\section{PERÍNEO:}

$\left\{\begin{array}{l}\text { Íntegro } \\ \text { Roto }- \text { Grau de rotura }\end{array}\right.$

Presença de lesōes

\section{COLO:}

- Características macroscópicas:

- Teste de Schiller:

IMPRESSÃO GERAL (Postura quanto à participação na entrevista):

INTERCORRÊNCIAS GERAIS OBSERVADAS:

CONDUTA: 